\title{
No-Envy, Efficiency, and Collective Rationality
}

\author{
Norihito Sakamoto* \\ Hitotsubashi University \\ January, 2007 \\ This Version: August, 2011
}

\begin{abstract}
We consider the problem of a fair collective choice function (fair CCF) which maps each profile of extended preference orderings into the set of fair social states (the set consists of alternatives which are both Pareto efficient and envy-free) when such a set exists. Our main objective is to examine compatibility of fair social choices with collective rationality. We formulate desirable properties of collective rationality, and look for CCFs satisfying them. Next, we show that there is no CCF that satisfies most of the choice-consistency properties and a simple concept of fairness simultaneously. Moreover, we reveal that there exists no collective choice function that selects efficient and envy-free states cannot be rationalized by a social preference relation.
\end{abstract}

*Department of Economics, Hitotsubashi University, 2-1 Naka, Kunitachi, Tokyo, Japan, 1868601. E-mail: b111126u@r.hit-u.ac.jp; Tel: +81-42-580-8192; Fax: +81-42-580-8195. This paper is part of my Ph. D. Thesis. The author is deeply grateful to Kotaro Suzumura, Koichi Tadenuma, Shinji Yamashige, Kiwako Sakamoto, Peter Lambert, Ryoichi Nagahisa, Yukihiro Nishimura and Toyotaka Sakai, Marc Fleurbaey and two anonymous refrees for helpful comments and also thanks the 21st century Centers of Excellence (COE) project on "Normative Evaluation and Social Choice of Contemporary Economic Systems" for financial support. Any remaining errors are my responsibility. 


\section{Introduction}

The objective of this paper is to examine compatibility of fair social choices with collective rationality. Here we define fair social states as Pareto efficient and envy-free states. The concept of equity as no-envy is central among various equity concepts that have been proposed and studied in economics. However, as Fleurbaey (2008) pointed out, the no-envy test is an all-or-nothing notion and we often face the cases in which there are no fair allocations (Pazner and Schmeidler 1974; Fleurbaey 1994). Some studies have been trying to construct desirable rankings on the set of social states which are not necessarily fair, and show various possibility/impossibility results about social ranking rules or choice functions based on the concept of fairness. (Feldman and Kirman 1974; Varian 1976; Suzumura 1981a; 1981b; 1983; Clauduri 1986; Diamantaras and Thomson 1990; Tadenuma 1998; 2002; 2008; Fleurbaey 2008). Suzumura (1981a; 1981b) classifies consistency axioms of choice functions and examines whether choice functions that select all fair social states for each profile of preferences satisfy these consistency axioms. Then, he obtains largely negative results. Tadenuma (2002) analyzes theoretical consequences of combining the Pareto quasi-ordering and an equity ordering a la Feldman-Kirman (Feldman and Kirman 1974) lexicographically. He shows that lexicographic compositions of the Pareto quasi-ordering and the equity ordering violate acyclicity when the Pareto criterion is given priority over the equity criterion. Another example is the study by Fleurbaey and Trannoy (2003) that shows any social ranking based on equity as no-domination in commodities and Pareto quasi-orderings generates a cycle for some profiles of preference relations. ${ }^{1}$ These studies suggest that

\footnotetext{
${ }^{1}$ Equity as no-domination is a necessary condition for envy-freeness. Hence, the impossibility result showed by Fleurbaey and Trannoy (2003) also holds under the no-envy criterion.
} 
attempts to respect both Pareto efficiency and no-envy often result in contradiction with consistency axioms for collective choice.

This paper reexamines the results shown by these previous studies, and extends some of them. Specifically, this paper analyzes whether possibility results are obtainable by weakening the requirement of fairness. We consider the requirement that a subset of social states be chosen in the set of efficient and envy-free ones whenever the latter set is non-empty. This is weaker than the axiom "Fairness Extension" introduced and studied in Suzumura (1981a), which requires that the whole set of efficient and envy-free states be chosen. With this requirement on fairness, we rewrite the map of impossibility results shown by Suzumura (1981a). Moreover, we show non-existence of a choice function that satisfies the above axiom on fairness and Chernoff's Axiom, which is one of the most fundamental axioms for collective rationality. Since Chernoff's Axiom is a necessary condition for a choice function to have a rationalization, our impossibility result means that no choice function that selects efficient and envy-free states cannot be rationalized by a social preference relation.

The remainder of the paper is as follows. The following section explains the definitions and notations of basic terminology. Section 3 introduces axioms about consistency of collective choice and shows possibility/ impossibility results on collective rationality and our notion of fairness. Section 4 briefly discusses our results in economic environments. Finally, Section 5 comments on further extensions and implications of our results. 


\section{Basic Notations and Definitions}

Let $X$ and $\mathcal{S}$ stand, respectively, for the finite set of all conceivable social states and the set of all non-empty subsets of $X$. We call a set $S \in \mathcal{S}$ an opportunity set. The society consists of a finite set of individuals $N=\{1,2, \ldots, n\}$ where $|N|=n \geq 2$. Assume each individual $i \in N$ has an extended preference ordering $\tilde{R}_{i}$ on the Cartesian product $X \times N$, which is reflexive, connected and transitive. For any $(x, j),(y, k) \in X \times N$, $((x, j),(y, k)) \in \tilde{R}_{i}$ implies that being in the position of individual $j$ in the social state $x$ is at least as good as being in the position of individual $k$ in the social state $y$ according to $i$ 's view. We hereafter denote $((x, j),(y, k)) \in \tilde{R}_{i}$ as $(x, j) \tilde{R}_{i}(y, k)$ for all $(x, j),(y, k) \in X \times N$. Let $\tilde{P}_{i}$ and $\tilde{I}_{i}$ be the asymmetric part and the symmetric part of $\tilde{R}_{i}$ respectively. A profile of n-tuple extended preference orderings is indexed by $\tilde{R}_{N}=\left(\tilde{R}_{1}, \tilde{R}_{2}, \ldots, \tilde{R}_{n}\right), \tilde{R}_{N}^{\prime}=\left(\tilde{R}_{1}^{\prime}, \tilde{R}_{2}^{\prime}, \ldots, \tilde{R}_{n}^{\prime}\right)$, and so on. Let $\tilde{\mathcal{R}}_{I}$ be the set of all logically possible profiles which satisfy the axiom of identity (Sen 1970). The axiom of identity requires that an individual $i$ should respect an individual $j$ 's judgment on $(x, j)$ and $(y, j)$, that is,

$$
\forall x, y \in X, \forall i, j \in N ;(x, j) \tilde{R}_{i}(y, j) \Leftrightarrow(x, j) \tilde{R}_{j}(y, j)
$$

A collective choice function is a mapping $C: \mathcal{S} \times \tilde{\mathcal{R}}_{I} \rightarrow \mathcal{S}$ such that $C(S, R) \subseteq S$ for all $S \in \mathcal{S}$ and $\tilde{R}_{N} \in \tilde{\mathcal{R}}_{I}$. Since the range of collective choice functions is the set of non-empty subsets of $X$, collective choice functions are required to select some states for each and every choice situation.

Now, we introduce concepts of efficiency and equity. Given a profile $\tilde{R}_{N}=\left(\tilde{R}_{1}, \tilde{R}_{2}, \ldots, \tilde{R}_{n}\right) \in$ 
$\tilde{\mathcal{R}}_{I}$ and an opportunity set $S \in \mathcal{S}$, for each individual $i \in N$, let $i$ 's subjective preference ordering $R_{i}$ be defined by $R_{i}=\left\{(x, y) \in X \times X \mid(x, i) \tilde{R}_{i}(y, i)\right\}$. Next, let the Pareto quasi-ordering $\succsim_{P}$ be defined by $\succsim_{P}=\bigcap_{i \in N} R_{i}$. The set of all Pareto efficient states in $S \in \mathcal{S}$ is denoted by $P\left(S, \tilde{R}_{N}\right)$, that is, $P\left(S, \tilde{R}_{N}\right)=\left\{x \in S \mid \forall y \in S, y \nsucc_{P} x\right\}$, where $\succ_{P}$ denotes the asymmetric part of $\succsim_{P}$.

According to the classical definition of Foley (1967) and Kolm (1972), an individual $i \in N$ envies an individual $j \in N$ at $x \in X$ if and only if $(x, j) \tilde{P}_{i}(x, i)$ holds. A state $x$ is said to be envy-free if and only if nobody envies other individuals at $x$. The set of all envy-free states in $S \in \mathcal{S}$ is denoted by $E\left(S, \tilde{R}_{N}\right)$.

If a state in $S$ is simultaneously Pareto efficient and envy-free, the state is said to be fair in $S$. The set of all fair states in $S$, called the fair set in $S$, is written as follows:

$$
F\left(S, \tilde{R}_{N}\right)=P\left(S, \tilde{R}_{N}\right) \cap E\left(S, \tilde{R}_{N}\right)
$$

Our objective is to investigate the existence and nature of fair collective choice functions, which assigns the set of fair states whenever such a set exists. The following explains a basic requirement of fair CCFs on which we focus in this paper.

\section{Fairness Extension (FE):}

$$
\forall \tilde{R}_{N} \in \tilde{\mathcal{R}}_{I}, \forall S \in \mathcal{S} ; F\left(S, \tilde{R}_{N}\right) \neq \emptyset \Rightarrow C\left(S, \tilde{R}_{N}\right)=F\left(S, \tilde{R}_{N}\right)
$$

Suzumura (1981a) introduces the above axiom and shows many variations of choiceconsistency are incompatible with FE. Thus, we formulate a weaker concept of fairness. 
Fairness Subset Extension (FSE) $:^{2}$

$$
\forall \tilde{R}_{N} \in \tilde{\mathcal{R}}_{I}, \forall S \in \mathcal{S} ; F\left(S, \tilde{R}_{N}\right) \neq \emptyset \Rightarrow C\left(S, \tilde{R}_{N}\right) \subseteq F\left(S, \tilde{R}_{N}\right)
$$

This axiom requires that, for all choice situations, socially chosen states be fair when such states exist. Clearly, the axiom FE implies FSE. Our axiom focuses only on situations where fair states exist, and these axioms therefore allow us to pick any social states from an opportunity set when no fair state exists.

\section{$3 \quad$ Fundamental Conflicts between Choice-Consistency}

\section{and Fairness}

In this section, we examine compatibility of fair collective choice functions with collective rationality. Rationality axioms of collective choice have been investigated by many previous studies in terms of Arrovian social choice theory. Following Suzumura's sophisticated taxonomy (Suzumura 1981a; 1981b), we consider nine axioms of collective rationality ${ }^{3}$.

First, we introduce Arrow's axiom which plays a major role in the context of "rationalizability of choice functions." As a preliminary step, we define the rationalizability of choice functions. A choice function $C$ is a mapping from $\mathcal{S}$ to $\mathcal{S}$ such that $C(S) \subset S$

\footnotetext{
${ }^{2}$ Denicolo (1999) calls this axiom "Weak Fairness Extension" and analyzes whether the requirement of fairness is consistent with informational efficiency such as IIA. In this paper, the name of "Fairness Extension" means a functional extension so we call this axiom "Fairness Subset Extension."

${ }^{3}$ Except for Arrow's Axiom, Chernoff's Axiom, Path-Independence, and Weak Path-Independence $\alpha$ and $\beta$, the following axioms are proposed and scrutinized by Suzumura (1981a; 1981b; 1983).
} 
for all $S \in \mathcal{S}$. Then, a choice function is fully rational, if there exists an ordering $R^{*}$ on $X$ such that

$$
C(S)=\left\{x \in S \mid \nexists y \in S, y P\left(R^{*}\right) x\right\} \text { for all } S \in \mathcal{S},
$$

where $P\left(R^{*}\right)$ is the asymmetric part of $R^{*}$.

That is, a choice function $C$ is fully rational if we can construct an ordering on $X$, and the set of maximal elements of this ordering equals the chosen set of the choice function for each and every opportunity set. Then, we call this ordering $R^{*}$ "a full rationalization of $C$ " and this choice function $C$ " a fully rationalizable choice function." In a similar way, if we can find a reflexive and complete binary relation and the set of maximal elements of the binary relation always equals a chosen set of a choice function, then this binary relation is called "a rationalization of the choice function" or "a binary relation rationalizing the choice function." Arrow (1959) proves that the following axiom is a necessary and sufficient condition for choice functions to be fully rational.

\section{Arrow's Axiom (AA):}

$$
\forall S_{1}, S_{2} \in \mathcal{S} ; S_{1} \subset S_{2} \Rightarrow\left[S_{1} \cap C\left(S_{2}\right)=\emptyset \text { or } \quad S_{1} \cap C\left(S_{2}\right)=C\left(S_{1}\right)\right]
$$

This axiom can be divided into two axioms formally. One is Chernoff's Axiom (Chernoff 1954) which is a necessary condition for a choice function to be rationalized by a binary relation. Thus, if a choice function does not satisfy Chernoff's Axiom, 
then there exists no binary relation rationalizing this function ${ }^{4}$.

\section{Chernoff's Axiom (CA):}

$$
\forall S_{1}, S_{2} \in \mathcal{S} ; S_{1} \subset S_{2} \Rightarrow S_{1} \cap C\left(S_{2}\right) \subset C\left(S_{1}\right)
$$

The other weak version of Arrow's Axiom is called Dual Chernoff's Axiom.

\section{Dual Chernoff's Axiom (DCA):}

$$
\forall S_{1}, S_{2} \in \mathcal{S} ; S_{1} \subset S_{2} \Rightarrow\left[S_{1} \cap C\left(S_{2}\right)=\emptyset \text { or } S_{1} \cap C\left(S_{2}\right) \supset C\left(S_{1}\right)\right]
$$

The next axiom is a well-known one, called "Path-Independence" which is introduced by Arrow (1963) and Plott (1973). This axiom requires that final outcomes chosen by any successive choice situations should be independent of the order of choice situations.

\section{Path-Independence (PI):}

$$
\forall S_{1}, S_{2} \in \mathcal{S} ; C\left(S_{1} \cup S_{2}\right)=C\left(C\left(S_{1}\right) \cup S_{2}\right)
$$

Following Ferejohn and Grether (1977), we divide Path-Independence into two axioms.

\section{Weak Path-Independence $\alpha$ (WPI- $\alpha)$ :}

$$
\forall S_{1}, S_{2} \in \mathcal{S} ; C\left(S_{1} \cup S_{2}\right) \subset C\left(C\left(S_{1}\right) \cup S_{2}\right) .
$$

\footnotetext{
${ }^{4}$ See Tadenuma (2008, p.125). The author thanks an associate editor for the helpful remarks on the property of Chernoff's Axiom.
} 
Weak Path-Independence $\beta$ (WPI- $\beta)$ :

$$
\forall S_{1}, S_{2} \in \mathcal{S} ; C\left(S_{1} \cup S_{2}\right) \supset C\left(C\left(S_{1}\right) \cup S_{2}\right)
$$

Now, we proceed to the following axiom which is used in an axiomatic characterization of the Nash bargaining solution. By using an analogy of Sen's argument (1970, Ch.1*), this axiom can be explained as follows: if members of the best judo team of the world are all Japanese, then the best judo team in Japan must be the best team of the world.

\section{Nash's Axiom (NA):}

$$
\forall S_{1}, S_{2} \in \mathcal{S} ;\left[S_{1} \subset S_{2} \& C\left(S_{2}\right) \subset S_{1}\right] \Rightarrow C\left(S_{1}\right)=C\left(S_{2}\right)
$$

The remaining two axioms are Stability Axiom and Superset Axiom which are minimal requirements of choice consistency in this paper.

Stability Axiom (ST):

$$
\forall S \in \mathcal{S} ; C(S)=C(C(S))
$$

\section{Superset Axiom (SUA):}

$$
\forall S_{1}, S_{2} \in \mathcal{S} ;\left[S_{1} \subset S_{2} \& C\left(S_{2}\right) \subset C\left(S_{1}\right)\right] \Rightarrow C\left(S_{1}\right)=C\left(S_{2}\right)
$$

To make our argument easier, logical relations among the nine axioms above are 
summarized in the following proposition. Here an arrow indicates a logical implication which cannot be reversed in general.

\section{Proposition 1.}

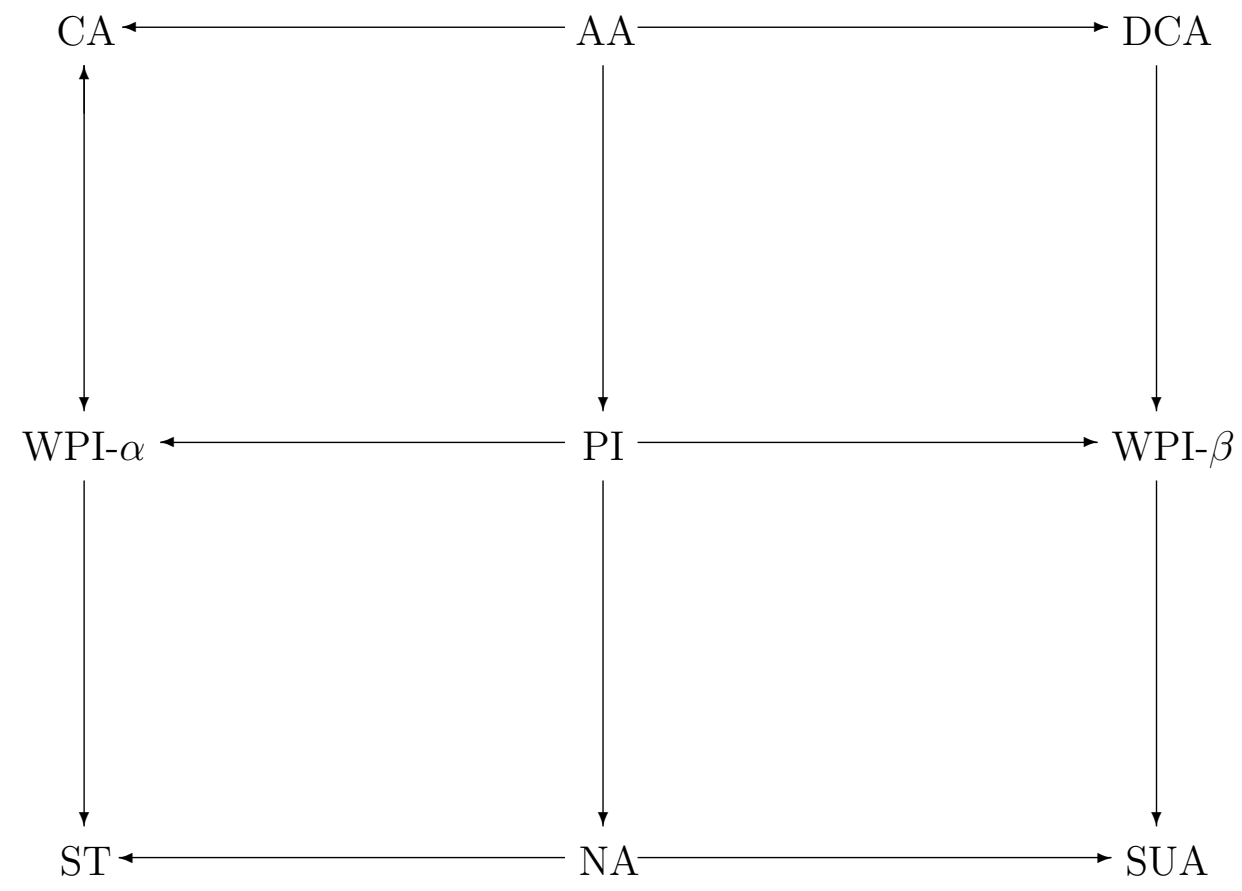

Proof. Most of the assertions can be directly induced by definitions or be already proved in Blair et al . (1976), Ferejohn and Grether (1977), Plott (1973) and Suzumura (1981a; 1983, Ch.2). Therefore, we only have to prove that PI implies NA.

Let a choice function $C$ satisfy PI. Then, for all $S_{1}, S_{2} \in \mathcal{S}$ such that $S_{1} \subset S_{2}$ and $C\left(S_{2}\right) \subset S_{1}$, it is trivial that $S_{1} \cup S_{2}=S_{2}$ and $S_{1} \cup C\left(S_{2}\right)=S_{1}$ hold. Thus, PI implies that $C\left(S_{2}\right)=C\left(S_{1} \cup S_{2}\right)=C\left(S_{1} \cup C\left(S_{2}\right)\right)=C\left(S_{1}\right)$. \|

Though these axioms are defined in the framework of simple choice functions, we can easily extend these consistency axioms to ones of a class of collective choice functions in the previous argument. Hereafter, we apply these axioms to collective choice functions in our framework. 
Now, let us examine the logical relationships between Fairness Subset Extension and collective rationality axioms. The first result shows that there exists no fair CCF satisfying Nash's Axiom.

Proposition 2. Suppose $|X| \geq 4$. Then, there exists no CCF which satisfies FSE (Fairness Subset Extension) and NA (Nash's Axiom).

Proof. Suppose to the contrary. Let a collective choice function $C$ satisfy FSE and NA. Take four distinct social states $x, y, z$ and $w$ and let $S=\{x, y, z, w\}$. Let a profile $\tilde{R}_{N}=\left(\tilde{R}_{1}, \tilde{R}_{2}, \ldots, \tilde{R}_{n}\right)$ be such that

$$
\begin{aligned}
& \left.\tilde{R}_{1}\right|_{S \times\{1,2\}}:(z, 1),(w, 2),(x, 1),(w, 1),(y, 1),(y, 2),(z, 2),(x, 2), \\
& \left.\tilde{R}_{2}\right|_{S \times\{1,2\}}:(w, 2),(z, 1),(y, 2),(z, 2),(x, 2),(x, 1),(w, 1),(y, 1),{ }^{5} \\
& \left.\tilde{R}_{i}\right|_{S \times\{1,2\}}=\left.\tilde{R}_{1}\right|_{S \times\{1,2\}} \quad \text { for all } i \in N \backslash\{1,2\},
\end{aligned}
$$

where $\left.\tilde{R}_{j}\right|_{S \times\{1,2\}}$ is the restriction of $\tilde{R}_{j}$ on $S \times\{1,2\}$ for all $j \in N$, and that

$$
\begin{aligned}
& \forall(v, j) \in(X \times N) \backslash(S \times\{1,2\}) ; \\
& \left\{\begin{array}{l}
(x, 2) \tilde{P}_{1}(v, j), \\
(y, 1) \tilde{P}_{2}(v, j), \\
(v, j) \tilde{P}_{i}(z, 1) \text { for all } i \in N \backslash\{1,2\},
\end{array}\right. \\
& \forall i \in N, \forall(v, j),(u, k) \in(X \times N) \backslash(S \times\{1,2\}) ;(v, j) \tilde{I}_{i}(u, k) .
\end{aligned}
$$

It is easy to show that this profile satisfies the axiom of identity. Clearly, $E\left(S, \tilde{R}_{N}\right)=$ $\{x, y\}, z \succ_{P} x$ and $w \succ_{P} y$ hold. Hence, we obtain $F\left(\{x, y, z\}, \tilde{R}_{N}\right)=\{y\}$ and

\footnotetext{
${ }^{5}$ Preference orderings are written in a decreasing order from left to right according to the degree of preference, i.e., if an alternative $A$ is preferred to an alternative $B, A$ must be on the left side of $B$.
} 
$F\left(\{x, y, w\}, \tilde{R}_{N}\right)=\{x\}$. By FSE, $C\left(\{x, y, z\}, \tilde{R}_{N}\right)=\{y\}$ and $C\left(\{x, y, w\}, \tilde{R}_{N}\right)=\{x\}$ must be true. Then, NA implies $C\left(\{x, y, z\}, \tilde{R}_{N}\right)=C\left(\{x, y\}, \tilde{R}_{N}\right)$ because of $\{x, y\} \subset$ $\{x, y, z\}$ and $C\left(\{x, y, z\}, \tilde{R}_{N}\right) \subset\{x, y\}$. Similarly, NA implies $C\left(\{x, y, w\}, \tilde{R}_{N}\right)=$ $C\left(\{x, y\}, \tilde{R}_{N}\right)$ because of $\{x, y\} \subset\{x, y, w\}$ and $C\left(\{x, y, w\}, \tilde{R}_{N}\right) \subset\{x, y\}$. However, it is impossible that $C\left(\{x, y, z\}, \tilde{R}_{N}\right)=C\left(\{x, y\}, \tilde{R}_{N}\right)=C\left(\{x, y, w\}, \tilde{R}_{N}\right)$ by the above argument, so we have the desirable contradiction. \|

Second, we derive an impossibility result about fair CCFs satisfying WPI- $\beta$.

Proposition 3. Suppose $|X| \geq 4$. Then, there exists no CCF which satisfies FSE (Fairness Subset Extension) and WPI- $\beta$ (Weak Path-Independence $\beta$ ).

Proof. Suppose to the contrary. Let a collective choice function $C$ satisfy FSE and WPI- $\beta$. Take four distinct social states $x, y, z$ and $w$ and let $S=\{x, y, z, w\}$. Consider the profile $\tilde{R}_{N}=\left(\tilde{R}_{1}, \tilde{R}_{2}, \ldots, \tilde{R}_{n}\right)$ in the proof of Proposition 2 .

By WPI- $\beta$, we have $C\left(\{x, y, z\}, \tilde{R}_{N}\right) \supset C\left(\{x\} \cup C\left(\{y, z\}, \tilde{R}_{N}\right), \tilde{R}_{N}\right)$. Then, the nonemptiness of CCF and $C\left(\{x, y, z\}, \tilde{R}_{N}\right)=\{y\}$ imply $C\left(\{x\} \cup C\left(\{y, z\}, \tilde{R}_{N}\right), \tilde{R}_{N}\right)=\{y\}$. Because FSE means $C\left(\{y, z\}, \tilde{R}_{N}\right)=\{y\}$, we obtain $C\left(\{x\} \cup C\left(\{y, z\}, \tilde{R}_{N}\right), \tilde{R}_{N}\right)=$ $\{y\}=C\left(\{x, y\}, \tilde{R}_{N}\right)$.

Next, consider $C\left(\{x, y, w\}, \tilde{R}_{N}\right)$. By WPI- $\beta$, we have $C\left(\{x, y, w\}, \tilde{R}_{N}\right) \supset C\left(C\left(\{x, y\}, \tilde{R}_{N}\right) \cup\right.$ $\left.\{w\}, \tilde{R}_{N}\right)$. Then, the non-emptiness of CCF and $C\left(\{x, y, w\}, \tilde{R}_{N}\right)=\{x\} \operatorname{imply} C\left(C\left(\{x, y\}, \tilde{R}_{N}\right) \cup\right.$ $\left.\{w\}, \tilde{R}_{N}\right)=\{x\}$. However, it must be that $C\left(\{x, y\}, \tilde{R}_{N}\right)=\{y\}$ by the above argument. Hence, we have $C\left(C\left(\{x, y\}, \tilde{R}_{N}\right) \cup\{w\}, \tilde{R}_{N}\right)=C\left(\{y, w\}, \tilde{R}_{N}\right)$ and $x \notin$ $C\left(C\left(\{x, y\}, \tilde{R}_{N}\right) \cup\{w\}, \tilde{R}_{N}\right)$. A contradiction. $\|$

Suzumura (1981a) proves that FE is incompatible with SUA which is weaker than 
NA and WPI- $\beta$.

Proposition 4. (Suzumura 1981a, Th.2) Suppose $|X| \geq 3$. Then, there exists no CCF which satisfies FE (Fairness Extension) and SUA (Superset Axiom).

However, we obtain the following possibility result when FE is weakened to FSE.

Proposition 5. There exist CCFs which satisfy FSE (Fairness Subset Extension), SUA (Superset Axiom) and ST (Stability Axiom).

Proof. As a preliminary step, let $\pi$ be a bijection from $X$ to $\{1,2, \ldots,|X|\}$. Then, we define a $\pi$-fair collective choice function $C_{\pi}$ as follows: for all $S \in \mathcal{S}$ and $\tilde{R}_{N} \in \tilde{\mathcal{R}}_{I}$,

$$
C_{\pi}\left(S, \tilde{R}_{N}\right)= \begin{cases}\arg \min _{x \in F\left(S, \tilde{R}_{N}\right)} \pi(x) & \text { if } F\left(S, \tilde{R}_{N}\right) \neq \emptyset \\ \arg \min _{x \in P\left(S, \tilde{R}_{N}\right)} \pi(x) & \text { otherwise. }\end{cases}
$$

Obviously, the $\pi$-fair collective choice function $C_{\pi}$ satisfies FSE and ST by definition. Moreover, it satisfies SUA because of single-valuedness of $\pi$-fair CCF. ॥

Proposition 5 shows that FSE is not only consistent with SUA but also consistent with ST. In the remainder of this paper, we will show that FE is compatible with ST. However, we may find these possibility results unacceptable because fair CCFs violate some basic choice-consistency properties such as Path-Independence. In fact, the next proposition implies that it is difficult to construct a fair CCF which satisfies a desirable choice-consistency property.

Proposition 6. Suppose $|X| \geq 4$. Then, there exists no CCF which satisfies FSE (Fairness Subset Extension) and CA (Chernoff's Axiom).

Proof. Suppose to the contrary. Let a collective choice function $C$ satisfy FSE and 
CA. Take four distinct social states $x, y, z$ and $w$ and let $S=\{x, y, z, w\}$. Consider the profile $\tilde{R}_{N}=\left(\tilde{R}_{1}, \tilde{R}_{2}, \ldots, \tilde{R}_{n}\right)$ in the proof of Proposition 2 .

Then, we have $C\left(\{x, y, z\}, \tilde{R}_{N}\right)=\{y\}$ and $C\left(\{x, y, w\}, \tilde{R}_{N}\right)=\{x\}$ by virtue of FSE. Consider now $C\left(\{x, y, z, w\}, \tilde{R}_{N}\right)$. If $y \in C\left(S, \tilde{R}_{N}\right)$ or $w \in C\left(S, \tilde{R}_{N}\right)$ holds, then it contradicts the fact that $C\left(\{x, y, w\}, \tilde{R}_{N}\right)$ equals a singleton set $\{x\}$ by virtue of CA. Therefore we have $y, w \notin C\left(S, \tilde{R}_{N}\right)$. Similarly, we induce $x, z \notin C\left(S, \tilde{R}_{N}\right)$ by CA and $C\left(\{x, y, z\}, \tilde{R}_{N}\right)=\{y\}$. Hence, it follows that $x, y, z, w \notin C\left(\{x, y, z, w\}, \tilde{R}_{N}\right)$, but this violates the non-emptiness of CCF. $\|$

Proposition 6 shows that fair CCFs are incompatible with Chernoff's Axiom. Since Chernoff's Axiom is a necessary condition for constructing a rationalization of CCF, there exists no social preference relation rationalizing a CCF which assigns a subset of fair states on each and every opportunity set whenever fair states exist.

Now, we compare our results with previous findings in Suzumura (1981a) and Tadenuma (1998; 2002). Suzumura (1981a) shows that the axioms "Fairness Inclusion (FI)" and "Conditional Binary Exclusion Pareto (CBEP)" are incompatible with Chernoff's Axiom but he doesn't say anything about the logical consequence of combining FE and $\mathrm{CA}$ on a $\mathrm{CCF}^{6}$. Since the axiom FSE implies FE, Proposition 6 implies that there exists no CCF satisfying both FE and CA. On the contrary, Suzumura's impossibility result fails to show an essential conflict between the concept of fairness and collective rationality, because both FI and CBEP imply that a socially chosen alternatives are not necessarily fair.

\footnotetext{
${ }^{6}$ The axiom FI is a requirement defined as follows: Suppose $x, y \in S$ and $y \in C(S)$. If $x$ is more efficient (or equitable) than $y$, then $x$ should be also in $C(S)$. On the other hand, CBEP requires that $x$ be the only chosen state in $\{x, y\}$ whenever both $x$ and $y$ are not envy-free and $x \succ_{P} y$.
} 
On the other hand, Tadenuma (2002) proves that his so-called "Efficiency-First Rule" -if two alternatives are Pareto comparable, then society respects the Pareto relation; otherwise social ranking should be ordered according to the equity criterionhas a cycle for some cases. In this lexicographic ranking, the set of maximal elements are the set of all Pareto efficient and envy-free allocations whenever fair allocations exist. Moreover, Tadenuma (1998) proves that any CCF which satisfies Pareto Efficiency, P-Conditional No-Envy, and Chernoff's Axiom violates the non-emptiness of CCF. Pareto Efficiency requires that CCFs should assign a subset of Pareto efficient allocations and P-Conditional No-Envy demands that a socially chosen set should be included in a subset of envy-free allocations if all the available allocations are efficient. Then, it is easy to show that CCFs satisfying Pareto Efficiency, P-Conditional NoEnvy, and Chernoff's Axiom also satisfy FSE because these CCFs assign a subset of fair allocations whenever they exist. Therefore, Proposition 6 is a generalization of Tadenuma's finding in the sense that a class of collective choice functions satisfying FSE and Chernoff's Axiom violates the non-emptiness of CCF.

Finally, we show that FE and ST are consistent but in a trivial result.

Proposition 7. There exist CCFs which satisfy FE (Fairness Extension) and ST (Stability Axiom).

Proof. Consider the Goldman-Sussangkarn (Goldman and Sussangkarn 1978) fair CCF $C_{G S}$ defined as follows ${ }^{7}: \forall S \in \mathcal{S}, \forall \tilde{R}_{N} \in \tilde{\mathcal{R}}_{I}$

\footnotetext{
${ }^{7}$ This CCF was proposed by Suzumura (1981b).
} 
$C_{G S}\left(S, \tilde{R}_{N}\right)= \begin{cases}F\left(S, \tilde{R}_{N}\right) & \text { if } F\left(S, \tilde{R}_{N}\right) \neq \emptyset \\ E(\bullet) \cup\left\{x \in P(\bullet) \mid \forall y \in E(\bullet), x \succ_{P} y\right\} & \text { if } E(\bullet) \neq \emptyset \& F(\bullet)=\emptyset \\ P\left(S, \tilde{R}_{N}\right) & \text { otherwise. }\end{cases}$

Or we define the Efficiency-First CCF $C_{P E}$ such that:

$\forall S \in \mathcal{S}, \forall \tilde{R}_{N} \in \tilde{\mathcal{R}}_{I}$

$C_{P E}\left(S, \tilde{R}_{N}\right)= \begin{cases}E\left(P\left(S, \tilde{R}_{N}\right), \tilde{R}_{N}\right) & \text { if } E\left(P\left(S, \tilde{R}_{N}\right), \tilde{R}_{N}\right) \neq \emptyset \\ P\left(S, \tilde{R}_{N}\right) & \text { otherwise. }\end{cases}$

It is easy to verify that both CCFs satisfy FE and ST. ॥

Propositions 1-7 reveal conflicts between collective rationality and our requirement of fairness, and we can summarize these results as follows.

Theorem 1. Suppose $|X| \geq 4$. Let CCF satisfy FSE (Fairness Subset Extension). Then, there exists no CCF satisfying any of the following consistency axioms: AA (Arrow's Axiom), CA (Chernoff's Axiom), DCA (Dual Cernoff's Axiom), PI (Path Independence), WPI- $\alpha$ (Weak Path Independence- $\alpha$ ), WPI- $\beta$ (Weak Path Independence- $\beta$ ), and NA (Nash's Axiom).

\section{Impossibility Results in Economic Environments}

This section shows that the previous results obtained in abstract environments also hold true in economic environments. In the previous section, we assume each profile of extended preference relations satisfies the axiom of identity. In this section, however, we suppose that all preference relations are monotonic and continuous. 
We consider the classical model of a division economy with $n$ individuals and $l$ goods. Let allocation be a vector $x=\left(x_{1}, x_{2}, \ldots, x_{n}\right) \in R_{+}^{n l}$ where each $x_{i}=$ $\left(x_{i 1}, \ldots, x_{i l}\right) \in R_{+}^{l}$ is a consumption bundle of individual $i \in N$. In this setting of economic environments, we assume that $X=R_{+}^{n l}$ and denote $\mathcal{S}$ by the set of all finite non-empty subsets of $X$. Each individual is assumed to have a preference ordering on his/her consumption bundle which is monotonic and continuous. Let $R_{E}$ be the set of all preference relations on $R_{+}^{l}$ that satisfy reflexivity, connectedness, transitivity, monotonicity and continuity. We write the set of profiles of preference orderings satisfying monotonicity and continuity as $\mathcal{R}_{E}$.

Then, we can redefine the set of Pareto efficient allocations and the set of envy-free allocations as follows: For all $S \in \mathcal{S}, R_{N} \in \mathcal{R}_{E}$,

$$
\begin{aligned}
& P\left(S, R_{N}\right):=\left\{x \in S \mid \nexists y \in S,\left[\forall i \in N, y_{i} R_{i} x_{i} \text { and } \exists j \in N, y_{j} P_{j} x_{j}\right\}\right. \\
& E\left(S, R_{N}\right):=\left\{x \in S \mid \forall i, j \in N, x_{i} R_{i} x_{j}\right\} .
\end{aligned}
$$

The axioms of FSE and choice-consistency in economic environments readily follow from the ones in the previous section; we omit the redefined statements of these requirements.

Now we show the existence of fair CCFs satisfying SUA and ST in economic environments.

Proposition 8. Suppose $X=R_{+}^{n l}$ and $\forall i \in N, R_{i} \in R_{E}$. Then, there exist CCFs which satisfy FSE (Fairness Subset Extension), SUA (Superset Axiom) and ST (Stability Axiom).

Proof. Consider a collective choice function $C_{L}$ as follows: for all $S$ and $R_{N}$, 
$C_{L}\left(S, R_{N}\right)= \begin{cases}\left\{x \in F\left(S, R_{N}\right) \mid \forall y \in F\left(S, R_{N}\right), x \geq_{L} y\right\} & \text { if } F\left(S, R_{N}\right) \neq \emptyset \\ \left\{x \in P\left(S, R_{N}\right) \mid \forall y \in P\left(S, R_{N}\right), x \geq_{L} y\right\} & \text { otherwise, }\end{cases}$

where a binary relation $\geq_{L}$ is a lexicographic order ${ }^{8}$.

Since the above CCF is a single-valued, it satisfies the axiom SUA. It is obvious that this CCF also satisfies both FSE and ST by definition. $\|$

Next we prove the axiom FSE is inconsistent with most of the choice-consistency axioms. As a preliminary step, we prove that a similar situation used in the proofs of Propositions 2, 3, and 6 also holds in economic environments.

Lemma 1 (Cf: Tadenuma 2002, Prop. 1). There exist a profile $R_{N} \in \mathcal{R}_{E}$ and four allocations $x, y, z, w \in X$ such that $E\left(\{x, y, z, w\}, R_{N}\right)=\{x, y\}, P\left(\{x, z\}, R_{N}\right)=$ $\{z\}, P\left(\{y, w\}, R_{N}\right)=\{w\}$ and $P\left(\{s, t\}, R_{N}\right)=\{s, t\}$ for all distinct pairs $\{s, t\}$ except for $\{x, z\}$ and $\{y, w\}$.

Proof. For simplicity, we consider a two-individuals and two-goods economy. Note that we can find similar examples for any pure exchange economies. Let an initial endowment be $(10,10)$. Consider four feasible allocations $x, y, z, w$ such that $x=((9,1),(1,9)), y=((8,2),(2,8)), z=((7,1.9),(3,8.1)), w=((6,2.9),(4,7.1))$. Suppose each individual has a preference relation $R_{i}(i=1,2)$ on $R_{+}^{2}$ that is represented by the following utility function:

$$
\begin{aligned}
& U_{1}\left(x_{11}, x_{12}\right)=x_{11} x_{12}, \\
& U_{2}\left(x_{21}, x_{22}\right)=x_{21}+2 x_{22} .
\end{aligned}
$$

\footnotetext{
${ }^{8} \mathrm{~A}$ Lexicographic order is defined as follows: For all $x, y \in X$, $x \geq_{L} y \Leftrightarrow \exists m>0, \forall i<m, x_{i}=y_{i} \& x_{m} \geq y_{m}$.
} 
Then, it follows $E\left(\{x, y, z, w\}, R_{N}\right)=\{x, y\}$ by definition. Since an allocation $z$ is Pareto superior to $x$ and so is $w$ to $y$, we have $P\left(\{x, z\}, R_{N}\right)=\{z\}$ and $P\left(\{y, w\}, R_{N}\right)=$ $\{w\}$. Finally, because all pairs $\{x, y\},\{x, w\},\{y, z\}$ and $\{z, w\}$ are Pareto noncomparable, we have $P\left(\{s, t\}, R_{N}\right)=\{s, t\}$ for all distinct pairs $\{s, t\}$ except for $\{x, z\}$ and $\{y, w\} . \|$

We can prove the following impossibility theorem by using Lemma 1 and proofs which are similar to that of Propositions 2, 3 and 6 in the last section. Hence, the impossibility theorem holds true in economic environments as well as in abstract environments.

Theorem 2. Suppose $X=R_{+}^{n l}$ and $\forall i \in N, R_{i} \in R_{E}$. Let CCF satisfy FSE (Fairness Subset Extension). Then, there exists no CCF satisfying any of the following consistency axioms: AA (Arrow's Axiom), CA (Chernoff's Axiom), DCA (Dual Cernoff's Axiom), PI (Path Independence), WPI- $\alpha$ (Weak Path Independence- $\alpha$ ), WPI- $\beta$ (Weak Path Independence- $\beta$ ), and NA (Nash's Axiom).

\section{Concluding Remarks}

This paper reexamines the relationships between collective rationality and the concept of fairness. We prove that there are CCFs satisfy FSE, SUA and ST. However, we show any fair CCF violates Chernoff's axiom, and thus there is no rationalization of a fair CCF.

Now it should be noted that our results are all based on a single-profile. In contrast to the multi-profile case, it holds without imposing any requirement of informational 
efficiency such as IIA. In addition, the same impossibility results holds true even if we require that a socially chosen set should be a subset of envy-free and weak Pareto efficient alternatives ${ }^{9}$.

Finally if we weaken Fairness Subset Extension sufficiently, we can construct fair CCFs satisfying some basic axioms of collective rationality such as PI. Consider the following axiom:

\section{Minimal Fairness ${ }^{10}$ :}

$$
\forall \tilde{R}_{N} \in \tilde{\mathcal{R}}_{I}, \forall S \in \mathcal{S} ; F\left(S, \tilde{R}_{N}\right) \neq \emptyset \Rightarrow C\left(S, \tilde{R}_{N}\right) \cap F\left(S, \tilde{R}_{N}\right) \neq \emptyset
$$

Then, the following $\mathrm{CCF} C_{E P}$ satisfies both the above fairness axiom and PI.

$$
C_{E P}\left(S, \tilde{R}_{N}\right)= \begin{cases}P\left(E\left(S, \tilde{R}_{N}\right), \tilde{R}_{N}\right) & \text { if } E\left(S, \tilde{R}_{N}\right) \neq \emptyset \\ P\left(S, \tilde{R}_{N}\right) & \text { otherwise. }\end{cases}
$$

Since the set $S \backslash E\left(S, \tilde{R}_{N}\right)$ could contain alternatives that are Pareto superior to the elements of $C_{E P}\left(S, \tilde{R}_{N}\right)$, we have $F\left(S, \tilde{R}_{N}\right) \subseteq C_{E P}\left(S, \tilde{R}_{N}\right)$ for all $S$ and $\tilde{R}_{N}$. Hence, this CCF $C_{E P}$ violates FSE but satisfies Minimal Fairness. In addition, it can be proved this CCF satisfies $\mathrm{PI}^{11}$. However, a class of CCFs satisfying Minimal Fairness includes a trivial CCF, which associates with each $\left(S, \tilde{R}_{N}\right)$ the set of all available alternatives. The trivial CCF makes no sense on the issues of distributive justice because a social preference relation rationalizing this function is a null preference,

\footnotetext{
${ }^{9} \mathrm{An}$ alternative is weak Pareto efficient if there is no other available alternative that makes all individuals strictly better off. Obviously, the set of weak Pareto efficient alternatives is included in the set of Pareto efficient ones.

${ }^{10}$ This axiom is proposed by an anonymous referee. The author is thankful for the helpful suggestions.

${ }^{11}$ The reader can also check that this $\mathrm{CCF}$ violates DCA.
} 
which is indifferent among all alternatives. Hence we need to consider additional axioms under the requirement of Minimal Fairness in order to rule out the trivial $\mathrm{CCF}$.

\section{References}

[1] Arrow, K. J. (1951, 1963) Social Choice and Individual Values, 1st ed. 1951, 2nd ed. 1963, New Haven: Yale University Press.

[2] Arrow, K. J. (1959) "Rational Choice Functions and Orderings," Economica, Vol. 26, pp. 121-127.

[3] Blair, D. H., G. Bordes, J.S. Kelly and K. Suzumura (1976) "Impossibility Theorems without Collective Rationality," Journal of Economic Theory, Vol. 13, pp. 361-379.

[4] Chauduri, A. (1986) "Some Implications of an Intensity Measure of Envy," Social Choice and Welfare, Vol. 3, pp. 255-270.

[5] Chernoff, H. (1954) "Rational Selection of Decision Functions," Econometrica, Vol. 22, pp. 422-443.

[6] Denicolo, V. (1999) "No-Envy and Arrow's Conditions," Social Choice and Welfare, Vol. 16, pp. 585-597.

[7] Diamantaras, D. and W. Thomson (1990) "A Refinement and Extension of the No-envy Concept," Economics Letters, Vol. 33, pp. 217-222. 
[8] Feldman A. M., and A. Kirman (1974) "Fairness and Envy," American Economic Review, Vol. 64, pp. 996-1005.

[9] Ferejohn, J. A. and D. M. Grether (1977) "Weak Path Independence," Journal of Economic Theory, Vol. 14, pp. 19-31.

[10] Fleurbaey, M. (1994) "On Fair Compensation," Theory and Decision, Vol. 36, pp. 277-307.

[11] Fleurbaey, M. (2008) "To Envy or To Be Envied? Refinements of the Envy Test for the Compensation Problem," in P. K. Pattanaik, K. Tadenuma, Y. Xu, and N. Yoshihara, [eds.] Rational Choice and Social Welfare: Theory and Applications: Essays in Honor of Kotaro Suzumura, Berlin: Springer-Verlag.

[12] Fleurbaey, M. and A. Trannoy (2003) “The Impossibility of a Paretian Egalitarian," Social Choice and Welfare, Vol. 21, pp. 243-263.

[13] Foley, D. (1967) "Resource Allocation and the Public Sector," Yale Economic Essays, Vol. 7, pp. 45-98.

[14] Goldman, S. M. and C. Sussangkarn (1978) "On the Concept of Fairness," Journal of Economic Theory, Vol. 19, pp. 210-216.

[15] Kolm, S-C. (1972) Justice et Equite, Paris: Editions du Centre National de la Recherche Scientifique.

[16] Pazner, E. and D. Schmeidler (1974) "A Difficulty in the Concept of Fairness," Review of Economic Studies, Vol. 41, pp. 441-443. 
[17] Plott, C. R. (1973) "Path Independence, Rationality, and Social Choice," Econometrica, Vol. 41, pp. 1075-1091.

[18] Sen, A. K. (1970) Collective Choice and Social Welfare, San Francisco: HoldenDay.

[19] Suzumura, K. (1981a) "On Pareto-Efficiency and the No-Envy Concept of Equity," Journal of Economic Theory, Vol. 25, pp. 367-379.

[20] Suzumura, K. (1981b) "On the Possibility of 'Fair' Collective Choice Rule," International Economic Review, Vol. 22, pp. 351-364.

[21] Suzumura, K. (1983) Rational Choice, Collective Decisions and Social Welfare, Cambridge: Cambridge University Press.

[22] Tadenuma, K. (1998) "Efficiency First or Equity First? Two Principles and Rationality of Social Choice," Discussion Paper Series No.1998-01, Hitotsubashi University.

[23] Tadenuma, K. (2002) "Efficiency First or Equity First? Two Principles and Rationality of Social Choice," Journal of Economic Theory, Vol. 104, pp. 462-472.

[24] Tadenuma, K. (2008) "Choice-Consistent Resolutions of the Efficiency-Equity Trade-Off," in P. K. Pattanaik, K. Tadenuma, Y. Xu, and N. Yoshihara, [eds.] Rational Choice and Social Welfare: Theory and Applications: Essays in Honor of Kotaro Suzumura, Berlin: Springer-Verlag.

[25] Varian, H. (1976) "Two Problems in the Theory of Fairness," Journal of Public Economics, Vol. 5, pp. 249-260. 